\title{
INTERVAL ESTIMATION FOR THE RATIO OF SCALE PARAMETERS AND FOR ORDERED SCALE PARAMETERS ${ }^{1}$
}

\author{
G. Iliopoulos and S. Kourouklis
}

Received: Revised version:

\begin{abstract}
Confidence intervals for the ratio of scale parameters are constructed in general families of distributions with nuisance (location) parameters. Each of these intervals has coverage probability at least as large as that of the standard minimum size (i.e., minimum ratio of endpoints) interval and, in addition, smaller size. Then analogous improved confidence intervals for the scale parameters subject to order restriction are derived. The method of construction is similar to that in Goutis and Casella [5], [6]. Examples are given and include the normal and exponential distributions as well as the inverse Gaussian distribution which is not a purely location-scale model. Applications to interval estimation of the error variance in variance components models are also discussed.
\end{abstract}

\section{Introduction}

There are many situations where data (measurements) are available from two sources for the same objective. For example, an experiment may be conducted by two laboratories using different methods or different measuring instruments. To effectively understand whether there is a difference in variablility between the two sources, both point and interval estimates of the ratio of the corresponding population variances are required. Furthermore, if the two populations are assumed to be normal, a confidence interval for the ratio of variances that contains one may indicate that a two sample t-test is applicable for testing equality of the respective means.

Nagata [23] derived intervals for the ratio of the variances of two normal populations that were better than the standard F-interval, by extending arguments of Stein [25]. In

\footnotetext{
${ }^{1}$ AMS 1991 subject classifications: $62 \mathrm{C} 99,62 \mathrm{~F} 25$

Key words and phrases: Decision theory, scale parameter, confidence interval, variance components
} 
Section 2, under suitable monotone likelihood ratio conditions, confidence intervals for the ratio $\sigma_{1} / \sigma_{2}$ of the scale parameters $\sigma_{1}$ and $\sigma_{2}$ of two independent populations with nuisance (location) parameters are constructed, which dominate the standard minimum ratio of endpoints interval in terms of both coverage probability and ratio of endpoints. The construction is based on a modification of Goutis and Casella's [5], [6] technique. Interestingly, in some typical cases, the computation of the improved intervals involves exactly the same algorithm that produces the standard interval (but with different input variables), see Remark 2.1. Thus, the new intervals are easy to use in practice. Examples are given and include the normal, exponential, and inverse Gaussian distributions. Numerical results are also presented to indicate the magnitude of the improvement over the standard interval.

We mention that the companion problem of (decision theoretic) point estimation of $\sigma_{1} / \sigma_{2}$ has been studied by several researchers, see Gelfand and Dey [3], Madi and Tsui [20], Kubokawa [13], Madi [19], Ghosh and Kundu [4], Kubokawa and Srivastava $[16]$.

In Section 3, interval estimation of $\sigma_{1}$ subject to the restriction that $\sigma_{1} \leq \sigma_{2}$ is considered. This restriction arises very naturally in several problems of practical interest. For instance, suppose that the lifetime of a component manufactured using an old method follows the exponential distribution with hazard rate $\sigma_{2}$ and the lifetime of the same component manufactured using a new technology follows the exponential distribution with hazard rate $\sigma_{1}$. It is then reasonable to expect that $\sigma_{1} \leq \sigma_{2}$. Using the same monotone likelihood ratio conditions and exploiting again Goutis and Casella's [5], [6] idea, confidence intervals for $\sigma_{1}$ are constructed which improve on the standard intervals, i.e., those that do not take into account the order restriction. As before, the improvement is both in coverage probability and ratio of endpoints. It is noted that in typical cases the computational difficulty of the improved intervals is essentially the same as that of their standard counterparts, see Remark 3.1. Thus, the new intervals share practical merit. The results apply, in particular, to the problem of interval estimation of the error variance in a balanced one way random effects model, and (for this model) the magnitude of the improvement over the standard interval is investigated numerically. Interval estimation of $\sigma_{2}$ subject to $\sigma_{1} \leq \sigma_{2}$ is also outlined.

We note that the book by Robertson, Wright, and Dykstra [24] contains a detailed account on order restricted inference. Early work on this subject traces back to van Eeden [26], [27], [28], [29], [30] and recent developments are contained in van Eeden [31]. Relevant work includes Kushary and Cohen [17], Kubokawa and Saleh [14], Hwang and Peddada [7], and Cohen and Sackrowitz [2].

An appendix, Section 4, contains some technical results. We close this section by mentioning that the technique of Goutis and Casella [5], [6] was also used by Iliopoulos and Kourouklis [9] for the construction of an improved interval for the generalized variance in a multivariate normal context. 


\section{Improved intervals for the ratio $\sigma_{1} / \sigma_{2}$}

Let $S_{1}, S_{2}, T_{1}, T_{2}$ be independent statistics such that $S_{i} / \sigma_{i}$ and $T_{i} / \sigma_{i}$ have densities

$$
g_{i}(x) I_{(0, \infty)}(x) \quad \text { and } \quad h_{i}\left(x ; \mu_{i}, \sigma_{i}\right) I_{\left(\lambda_{i}, \infty\right)}(x)
$$

respectively, where $\mu_{i}, \sigma_{i}$ are unknown parameters, $\sigma_{i}$ is positive and $\lambda_{i}=\lambda_{i}\left(\mu_{i}, \sigma_{i}\right)$ is a real valued function of $\mu_{i}$ and $\sigma_{i}, i=1,2$. The problem is to construct a "good" confidence interval for the ratio $\sigma=\sigma_{1} / \sigma_{2}$. For instance, if $X_{1}, \ldots, X_{n}$ and $Y_{1}, \ldots, Y_{m}$ are two independent random samples from exponential distributions $E\left(\mu_{1}, \sigma_{1}\right)$ and $E\left(\mu_{2}, \sigma_{2}\right)$ then $S_{1}=\sum_{i=1}^{n}\left(X_{i}-X_{(1)}\right), S_{2}=\sum_{i=1}^{m}\left(Y_{i}-Y_{(1)}\right), T_{1}=X_{(1)}=\min \left\{X_{i}: i=1, \ldots, n\right\}, T_{2}=$ $Y_{(1)}=\min \left\{Y_{i}: i=1, \ldots, m\right\}$ and $\lambda_{i}=\mu_{i} / \sigma_{i}$. See also Kubokawa [13] regarding the above framework.

We require the following condition.

(A1) $\frac{g_{i}\left(c_{1} x\right)}{g_{i}\left(c_{2} x\right)}$ is strictly increasing in $x>0$ for $0<c_{1}<c_{2}, i=1,2$.

We set $S=S_{1} / S_{2}$ and denote by $g(x), x>0$, the density of $S / \sigma$. Because of (A1), it follows from Lemmas 4.1 and 4.4 in the appendix that $g(x)$ is continuous and $x g(x)$ is unimodal. The standard confidence intervals for $\sigma$ have the form $I=(S / b, S / a), 0<$ $a<b$, and among them the one with confidence coefficient $1-\alpha$ and minimum ratio of endpoints is given by

$$
I_{0}=(S / b, S / a)
$$

where $a$ and $b$ uniquely satisfy

$$
\int_{a}^{b} g(x) d x=1-\alpha \quad \text { and } \quad a g(a)=b g(b) .
$$

We will need some further assumptions and a preliminary result. We suppose that there is a value $\left(\mu_{10}, \sigma_{10}\right)$ of $\left(\mu_{1}, \sigma_{1}\right)$ with $\lambda_{1}\left(\mu_{10}, \sigma_{10}\right)=0$ such that setting $h_{1}(x)=h_{1}\left(x ; \mu_{10}, \sigma_{10}\right), x>0$, the following two conditions hold.

(A2) $\frac{h_{1}\left(x ; \mu_{1}, \sigma_{1}\right) I_{\left(\lambda_{1}, \infty\right)}(x)}{h_{1}(x)}$ is nondecreasing in $x>0$.

(A3) $\frac{h_{1}\left(c_{1} x\right)}{h_{1}\left(c_{2} x\right)}$ is strictly increasing in $x>0$ for $0<c_{1}<c_{2}$.

Let $W_{1}=T_{1} / S_{1}$. Then, given $W_{1}=w_{1}>0$, the conditional density of $S / \sigma$ is

$$
f_{1}\left(x \mid w_{1} ; \mu_{1}, \sigma_{1}\right) \propto \int_{0}^{\infty} x v^{2} g_{1}(x v) h_{1}\left(x v w_{1} ; \mu_{1}, \sigma_{1}\right) g_{2}(v) I_{\left(\max \left(0, \lambda_{1}\right), \infty\right)}\left(x v w_{1}\right) d v .
$$

For the sake of simplicity, when $\mu_{1}=\mu_{10}$ and $\sigma_{1}=\sigma_{10}$ in (2.2) we write $f_{1}\left(x \mid w_{1}\right)$, that is

$$
f_{1}\left(x \mid w_{1}\right) \propto \int_{0}^{\infty} x v^{2} g_{1}(x v) h_{1}\left(x v w_{1}\right) g_{2}(v) d v .
$$

The following result is an application of Lemmas 4.1, 4.3, and 4.4. 
Lemma 2.1 (i) For $w_{1}>0$, under (A1) and (A3), we have that $f_{1}\left(x \mid w_{1}\right)$ is a continuous function of $x>0, x f_{1}\left(x \mid w_{1}\right)$ is a unimodal function of $x>0$, and $\lim _{x \rightarrow 0} x f_{1}\left(x \mid w_{1}\right)=$ $\lim _{x \rightarrow \infty} x f_{1}\left(x \mid w_{1}\right)=0$.

(ii) For $w_{1}>0$, under (A1) and (A2), $f_{1}\left(x \mid w_{1} ; \mu_{1}, \sigma_{1}\right) / f_{1}\left(x \mid w_{1}\right)$ is strictly increasing in $x>0$ unless the ratio in (A2) is constant as a function of $x>0$.

We now seek to improve on $I_{0}$ by constructing another interval that has smaller ratio of endpoints while maintaining the coverage probability above the nominal level $1-\alpha$. To motivate our choice of improved interval, we note that in typical cases (as, for instance, in Examples 1-3 below) the problem of interval estimation of $\sigma$ is invariant under the group of scale transformations $\left(T_{1}, S_{1}, T_{2}, S_{2}\right) \longrightarrow\left(c_{1} T_{1}, c_{1} S_{1}, c_{2} T_{2}, c_{2} S_{2}\right)$, $c_{i}>0, i=1,2$, and the corresponding class of scale equivariant intervals is given by $\left\{\left(\psi_{1}\left(W_{1}, W_{2}\right) S, \psi_{2}\left(W_{1}, W_{2}\right) S\right): \psi_{1}<\psi_{2}\right.$ positive functions $\}$, where $W_{2}=T_{2} / S_{2}$. A subclass which is simpler to study consists of intervals of the form

$$
I\left(S, W_{1}\right)=\left(\phi_{1}\left(W_{1}\right) S, \phi_{2}\left(W_{1}\right) S\right)
$$

for positive functions $\phi_{1}<\phi_{2}$. It is the latter class that we will next consider. Following Goutis and Casella [5], [6] we will require that $I\left(S, W_{1}\right)$ and $I_{0}$ have the same coverage probability when $\mu_{1}=\mu_{10}$ and $\sigma_{1}=\sigma_{10}$. Using Stein's [25] idea, this requirement will hold if it holds conditionally for each given value of $W_{1}$. Since we also seek to improve on the ratio of endpoints we will in addition require that $\phi_{2} / \phi_{1}$ is minimized. To this end, for each $w_{1}>0$, we determine $\phi_{10}=\phi_{10}\left(w_{1}\right)$ and $\phi_{20}=\phi_{20}\left(w_{1}\right)$ from the equations

$$
\int_{1 / \phi_{20}}^{1 / \phi_{10}} f_{1}\left(x \mid w_{1}\right) d x=\int_{a}^{b} f_{1}\left(x \mid w_{1}\right) d x \text { and } \frac{1}{\phi_{10}} f_{1}\left(\frac{1}{\phi_{10}} \mid w_{1}\right)=\frac{1}{\phi_{20}} f_{1}\left(\frac{1}{\phi_{20}} \mid w_{1}\right) .
$$

The existence and uniqueness of $\phi_{10}$ and $\phi_{20}$ follows from Lemma 2.1(i). Define now a confidence interval for $\sigma$ by

$$
I_{1}\left(S, W_{1}\right)=\left(\phi_{1}\left(W_{1}\right) S, \phi_{2}\left(W_{1}\right) S\right),
$$

where

$\phi_{1}\left(w_{1}\right)=\left\{\begin{array}{ll}\min \left\{\phi_{10}\left(w_{1}\right), 1 / b\right\} & , \text { if } w_{1}>0 \\ 1 / b & , \text { if } w_{1} \leq 0\end{array}\right.$ and $\phi_{2}\left(w_{1}\right)= \begin{cases}\min \left\{\phi_{20}\left(w_{1}\right), 1 / a\right\} & , \text { if } w_{1}>0 \\ 1 / a & , \text { if } w_{1} \leq 0 .\end{cases}$

The next theorem establishes the superiority of $I_{1}\left(S, W_{1}\right)$ over $I_{0}$. Condition (2.6) below is exemplified in Theorem 2.3. Also, the underlying value of $\left(\mu_{1}, \sigma_{1}\right)$ in the probability statements of the theorem is that specified in (A2).

Theorem 2.2 Assume that (A1), (A2), and (A3) hold. If

$$
\mathrm{P}\left(W_{1}>0 \text { and } b f_{1}\left(b \mid W_{1}\right)>a f_{1}\left(a \mid W_{1}\right)\right)>0
$$


then we have the following.

(i) $\mathrm{P}\left(\sigma \in I_{1}\left(S, W_{1}\right)\right) \geq \mathrm{P}\left(\sigma \in I_{0}\right)=1-\alpha$ and the inequality is strict unless the ratio in (A2) is constant as a function of $x>0$.

(ii) The ratio of endpoints of $I_{1}\left(S, W_{1}\right)$ is less than or equal to b/a (i.e., the ratio of endpoints of $I_{0}$ ) and is strictly less with positive probability.

Proof. (i) Let $B$ the set on which $I_{1}\left(S, W_{1}\right)$ differs from $I_{0}$, i.e., $B=\left\{w_{1}: w_{1}>\right.$ 0 and $\left.\phi_{10}\left(w_{1}\right)<1 / b\right\}$. We first show that $B=\left\{w_{1}: w_{1}>0\right.$ and $\left.b f_{1}\left(b \mid w_{1}\right)>a f_{1}\left(a \mid w_{1}\right)\right\}$. Let $w_{1}>0$ be such that

$$
b f_{1}\left(b \mid w_{1}\right)>a f_{1}\left(a \mid w_{1}\right) .
$$

Trivially, the first equality in (2.4) implies that neither of the intervals $\left(1 / \phi_{20}, 1 / \phi_{10}\right)$ and $(a, b)$ can be a proper subset of the other. Consequently, using the second equality in $(2.4),(2.7)$, and the unimodality of $x f_{1}\left(x \mid w_{1}\right)$ (Lemma 2.1 (i)), from Lemma 4.5 we conclude that $\phi_{10}\left(w_{1}\right)<1 / b$. Hence, $B \supseteq\left\{w_{1}: w_{1}>0\right.$ and $\left.b f_{1}\left(b \mid w_{1}\right)>a f_{1}\left(a \mid w_{1}\right)\right\}$. Conversely, consider $w_{1}>0$ with $\phi_{10}\left(w_{1}\right)<1 / b$. Then $a<b \leq 1 / \phi_{20}<1 / \phi_{10}$ or $a<1 / \phi_{20} \leq b<1 / \phi_{10}$. In either case, the unimodality of $x f_{1}\left(x \mid w_{1}\right)$ and (2.4) imply that $b f_{1}\left(b \mid w_{1}\right)>a f_{1}\left(a \mid w_{1}\right)$, and thus $B \subseteq\left\{w_{1}: w_{1}>0\right.$ and $\left.b f_{1}\left(b \mid w_{1}\right)>a f_{1}\left(a \mid w_{1}\right)\right\}$. To establish $(i)$, since the two intervals differ only on the set $B$ and $\mathrm{P}(B)>0$, by $(2.6)$, it suffices to show that

$$
\mathrm{P}\left(\sigma \in I_{1}\left(S, W_{1}\right) \mid W_{1}=w_{1}\right) \geq \mathrm{P}\left(\sigma \in I_{0} \mid W_{1}=w_{1}\right)
$$

for each $w_{1} \in B$ with strict inequality holding unless the ratio in (A2) is constant as a function of $x>0$. In the case of constant ratio in (A2), $f_{1}\left(x \mid w_{1} ; \mu_{1}, \sigma_{1}\right)=f_{1}\left(x \mid w_{1}\right)$ and hence we have equality in (2.8) for all $w_{1} \in B$ because of (2.4). Suppose now that the ratio in (A2) is not constant and consider $w_{1} \in B$. Then, (2.4), the relation $\phi_{10}\left(w_{1}\right)<$ $1 / b$, and the fact that $f_{1}\left(x \mid w_{1} ; \mu_{1}, \sigma_{1}\right) / f_{1}\left(x \mid w_{1}\right)$ is strictly increasing (Lemma 2.1 (ii)) imply $\int_{1 / \phi_{20}\left(w_{1}\right)}^{1 / \phi_{10}\left(w_{1}\right)} f_{1}\left(x \mid w_{1} ; \mu_{1}, \sigma_{1}\right) d x>\int_{a}^{b} f_{1}\left(x \mid w_{1} ; \mu_{1}, \sigma_{1}\right) d x$, by Lemma 4.6. Thus, (2.8) holds as strict inequality.

(ii) For a given value $w_{1}$ of $W_{1}$, the ratio of endpoints of $I_{1}\left(S, W_{1}\right)$ is either $b / a$ or $\phi_{20} / \phi_{10}$. In the latter case, $w_{1} \in B$ and we have $\phi_{20} / \phi_{10}<b / a$ since by construction $y=1 / \phi_{10}$ and $t=1 / \phi_{20}$ uniquely minimize $y / t$ subject to $\int_{t}^{y} f_{1}\left(x \mid w_{1}\right) d x=\delta$, where $\delta=\int_{a}^{b} f_{1}\left(x \mid w_{1}\right) d x$, see (2.4). The result now follows since $\mathrm{P}(B)>0$.

In a similar fashion we can construct an improved interval for $\sigma$ of the form

$$
I\left(S, W_{2}\right)=\left(\phi_{1}\left(W_{2}\right) S, \phi_{2}\left(W_{2}\right) S\right) .
$$

In this case, instead of (A2) and (A3), we need to suppose that there is a value $\left(\mu_{20}, \sigma_{20}\right)$ of $\left(\mu_{2}, \sigma_{2}\right)$ with $\lambda_{2}\left(\mu_{20}, \sigma_{20}\right)=0$ such that setting $h_{2}(x)=h_{2}\left(x ; \mu_{20}, \sigma_{20}\right), x>0$, the following two conditions hold. 


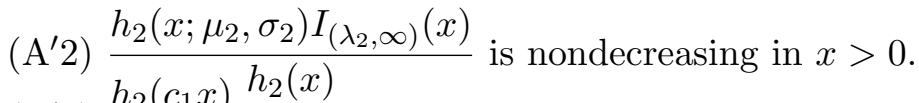

$\left(\mathrm{A}^{\prime} 3\right) \frac{h_{2}\left(c_{1} x\right)}{h_{2}\left(c_{2} x\right)}$ is strictly increasing in $x>0$ for $0<c_{1}<c_{2}$.

Details of the construction of $I\left(S, W_{2}\right)$ are given in Iliopoulos and Kourouklis [8].

The improved intervals in Theorem 2.2 and in (2.9) use only one of $T_{1}$ and $T_{2}$. It would be of considerable interest to construct improved intervals that use both of these statistics. The conditions in Theorem 2.2 are illustrated in the following general result.

Theorem 2.3 Suppose that $g_{i}(x)$ is a gamma density and that $\left(\mu_{10}, \sigma_{10}\right)$ in (A3) is such that $h_{1}(x)$ is also a gamma density, i.e., $g_{i}(x) \propto x^{\alpha_{i}-1} e^{-x / \beta_{i}}$ and $h_{1}(x) \propto x^{\gamma_{1}-1} e^{-x / \delta_{1}}, x>$ $0, \alpha_{i}, \beta_{i}, \gamma_{1}, \delta_{1}>0, i=1,2$. Then (A1), (A3), and (2.6) hold.

Proof. (A1) and (A3) are straightforward to show. Thus we only prove (2.6). Using (2.3) and the assumption of the theorem, it is easily seen that, for $w_{1}>0$, $b f_{1}\left(b \mid w_{1}\right)>a f_{1}\left(a \mid w_{1}\right)$ holds if and only if $w_{1}<w_{10}$, where $w_{10}$ is given by

$$
w_{10}=\delta_{1}\left\{\frac{1}{\beta_{2}} \frac{b^{d}-a^{d}}{b a^{d}-a b^{d}}-\frac{1}{\beta_{1}}\right\}
$$

and $d=\left(\alpha_{1}+\gamma_{1}\right) /\left(\alpha_{1}+\gamma_{1}+\alpha_{2}\right)$. Next, evaluating and substituting $g(x)$ in the second equation of (2.1) gives

$$
\frac{1 / \beta_{2}+b / \beta_{1}}{1 / \beta_{2}+a / \beta_{1}}=\left\{\frac{b}{a}\right\}^{\frac{\alpha_{1}}{\alpha_{1}+\alpha_{2}}} .
$$

Thus, from (2.10) and (2.11) we obtain that $w_{10}>0$, and consequently $\left\{w_{1}>0\right.$ : $\left.b f_{1}\left(b \mid w_{1}\right)>a f_{1}\left(a \mid w_{1}\right)\right\}=\left\{w_{1}: 0<w_{1}<w_{10}\right\}$. Since $W_{1}$ is supported on $(0, \infty)$ or $(-\infty, \infty)$ (depending on whether $\lambda_{1} \geq 0$ or $\left.\lambda_{1}<0\right)(2.6)$ holds.

Remark 2.1. We discuss some computational aspects of the interval $I_{1}\left(S, W_{1}\right)$ for the case considered in Theorem 2.3. First recall that for the computation of $I_{0}$ one needs to solve

$$
\int_{a}^{b} g\left(x ; \alpha_{1}\right) d x=1-\alpha \text { and } a g\left(a ; \alpha_{1}\right)=b g\left(b ; \alpha_{1}\right)
$$

for $a$ and $b$. Here $g\left(x ; \alpha_{1}\right)$ is the density of the ratio of two independent gamma densities, the notation stressing the dependency of the function $g$ on the shape parameter $\alpha_{1}$ of $g_{1}(x)$. Let $s$ and $w_{1}$ be the observed values of $S$ and $W_{1}$. Then $I_{1}\left(s, w_{1}\right) \neq I_{0}$ only if $0<w_{1}<w_{10}$. In that case, it is not difficult to show that $f_{1}\left(x \mid w_{1}\right) \propto g((1+$ $\left.\left.w_{1} \beta_{1} / \delta_{1}\right) x ; \alpha_{1}+\gamma_{1}\right)$. Next, because of $(2.4)$ and with $\epsilon=\int_{a\left(1+w_{1} \beta_{1} / \delta_{1}\right)}^{b\left(1+w_{1} \beta_{1} / \delta_{1}\right)} g\left(x ; \alpha_{1}+\gamma_{1}\right) d x$, to compute $I_{1}\left(s, w_{1}\right)$ one solves

$$
\int_{a^{*}}^{b^{*}} g\left(x ; \alpha_{1}+\gamma_{1}\right) d x=\epsilon \text { and } a^{*} g\left(a^{*} ; \alpha_{1}+\gamma_{1}\right)=b^{*} g\left(b^{*} ; \alpha_{1}+\gamma_{1}\right),
$$

for $a^{*}$ and $b^{*}$, evaluates $\phi_{10}=\left(1+w_{1} \beta_{1} / \delta_{1}\right) / b^{*}$ and $\phi_{20}=\left(1+w_{1} \beta_{1} / \delta_{1}\right) / a^{*}$, and delivers $I\left(s, w_{1}\right)=\left(\phi_{10} s, \phi_{20} s\right)$. We thus see that the main step in the computation of $I_{1}\left(S, W_{1}\right)$ 
is simply a rerun of the procedure that produces $I_{0}$ replacing $\alpha_{1}$ and $1-\alpha$ by $\alpha_{1}+\gamma_{1}$ and $\epsilon$ respectively. This makes the proposed interval $I_{1}\left(S, W_{1}\right)$ quite easy to use in practice.

Example 1. (Normal distribution) Let $U, V, S_{1}, S_{2}$ be independent random variables where $U \sim N_{p}\left(\mu_{1}, \sigma_{1}^{2} I_{p}\right), V \sim N_{q}\left(\mu_{2}, \sigma_{2}^{2} I_{q}\right), S_{1} / \sigma_{1}^{2} \sim \chi_{n}^{2}$ and $S_{2} / \sigma_{2}^{2} \sim \chi_{m}^{2}$. In this example the parameter of interest is $\sigma=\sigma_{1}^{2} / \sigma_{2}^{2}$. Accordingly, we have $T_{1}=\|U\|^{2}$ and $T_{2}=\|V\|^{2}$ with distributions $T_{1} / \sigma_{1}^{2} \sim \chi_{p}^{2}\left(\left\|\mu_{1}\right\|^{2} / \sigma_{1}^{2}\right)$ and $T_{2} / \sigma_{2}^{2} \sim \chi_{q}^{2}\left(\left\|\mu_{2}\right\|^{2} / \sigma_{2}^{2}\right)$ (so that $\lambda_{1}=\lambda_{2}=0$ ). Taking $\mu_{10}=0, \sigma_{10}^{2}=1$, Condition (A2) holds by the well known monotone likelihood ratio property of the noncentral chi-squared distribution. Also (A1), (A3), and (2.6) are satisfied by Theorem 2.3. Figures 1-3 give the coverage probability and percentage improvement in expected ratio of endpoints of $I_{1}\left(S, W_{1}\right)$ over the standard $F$-interval plotted against the noncentrality parameter $\lambda_{1}=\left\|\mu_{1}\right\|^{2} / \sigma_{1}^{2}$ for selected values of $n, m, p$ and nominal level $1-\alpha=0.95$. These numerical results indicate that for $\lambda_{1}$ close to zero and small values of $n, m$, the improvement in ratio of endpoints can be quite substantial, whereas the maximum coverage probability can reach 0.9549 .

Example 2. (Exponential distribution) Let $X_{1}, \ldots, X_{n}, n \geq 2$, and $Y_{1}, \ldots, Y_{m}$, $m \geq 2$, be two independent random samples from exponential distributions $E\left(\mu_{1}, \sigma_{1}\right)$ and $E\left(\mu_{2}, \sigma_{2}\right)$ with densities $\frac{1}{\sigma_{1}} e^{-\left(x-\mu_{1}\right) / \sigma_{1}} I_{\left(\mu_{1}, \infty\right)}(x)$ and $\frac{1}{\sigma_{2}} e^{-\left(x-\mu_{2}\right) / \sigma_{2}} I_{\left(\mu_{2}, \infty\right)}(x)$ respectively. Here the parameter of interest is $\sigma=\sigma_{1} / \sigma_{2}$, and $S_{1}=\sum_{i=1}^{n}\left(X_{i}-X_{(1)}\right), S_{2}=$ $\sum_{i=1}^{m}\left(Y_{i}-Y_{(1)}\right), T_{1}=X_{(1)}=\min \left\{X_{i}: i=1, \ldots, n\right\}$ and $T_{2}=Y_{(1)}=\min \left\{Y_{i}: i=1, \ldots, m\right\}$ with distributions $S_{1} / \sigma_{1} \sim \frac{1}{2} \chi_{2(n-1)}^{2}, S_{2} / \sigma_{2} \sim \frac{1}{2} \chi_{2(m-1)}^{2}, T_{1} / \sigma_{1} \sim E\left(\mu_{1} / \sigma_{1}, 1 / n\right)$ and $T_{2} / \sigma_{2} \sim E\left(\mu_{2} / \sigma_{2}, 1 / m\right)$ (so that $\lambda_{1}=\mu_{1} / \sigma_{1}$ and $\lambda_{2}=\mu_{2} / \sigma_{2}$ ). Taking $\mu_{10}=0, \sigma_{10}=1$, Condition (A2) holds trivially whereas (A1), (A3), and (2.6) are satisfied by Theorem 2.3 .

Example 3. (Inverse Gaussian distribution) Let $X_{1}, \ldots, X_{n}, n \geq 2$, and $Y_{1}, \ldots, Y_{m}$, $m \geq 2$, be two independent random samples from inverse Gaussian distributions with densities $\left(\frac{\sigma_{i}}{2 \pi x^{3}}\right)^{1 / 2} \exp \left\{-\frac{\sigma_{i}\left(x-\mu_{i}\right)^{2}}{2 \mu_{i}^{2} x}\right\}, x>0, \mu_{i}>0, \sigma_{i}>0, i=1,2$. In this example the parameter $\mu_{i}$ is not a location parameter and consequently this model is not a purely location-scale model. Here the parameter of interest is $\sigma=\sigma_{2} / \sigma_{1}\left(1 / \sigma_{i}\right.$ is often referred to as the dispersion parameter, so $\sigma$ is the ratio of dispersion parameters). The standard confidence interval, $I_{0}$, for $\sigma$ is based on $S=S_{1} / S_{2}$, where $S_{1}=\sum_{i=1}^{n}\left(\frac{1}{X_{i}}-\frac{1}{\bar{X}}\right)$, $S_{2}=\sum_{i=1}^{m}\left(\frac{1}{Y_{i}}-\frac{1}{\bar{Y}}\right)$, with distributions $\sigma_{1} S_{1} \sim \chi_{n}^{2}$ and $\sigma_{2} S_{2} \sim \chi_{m}^{2}$. To improve on $I_{0}$, we let $T_{1}=n(\bar{X}-1)^{2} / \bar{X}$ and $T_{2}=m(\bar{Y}-1)^{2} / \bar{Y}$ (so that $\lambda_{1}=\lambda_{2}=0$ ), and note that when $\mu_{i}=1, \sigma_{i} T_{i}$ has a $\chi_{1}^{2}$ distribution, $i=1,2$. Taking $\mu_{10}=1, \sigma_{10}=1$, Condition (A2) is shown to hold in Kourouklis [12], whereas (A1), (A3), and (2.6) are satisfied by Theorem 2.3. 


\section{Improved intervals for ordered scale parameters}

In this section we assume the model and the conditions of Section 2. Under the order restriction $\sigma_{1} \leq \sigma_{2}$ we address the problem of constructing better intervals for $\sigma_{1}$ than the standard ones, i.e., those that do not take into account this restriction. With $\sigma_{i 0}$, $i=1,2$, as in (A2), (A3), ( $\left.\mathrm{A}^{\prime} 2\right)$, and $\left(\mathrm{A}^{\prime} 3\right)$ we will need the following condition.

(C1) $\sigma_{10}=\sigma_{20}$.

The standard confidence interval for $\sigma_{1}$ with confidence coefficient $1-\alpha$ and minimum ratio of endpoints is given by

$$
I_{1}=\left(S_{1} / b_{1}, S_{1} / a_{1}\right)
$$

where $a_{1}$ and $b_{1}$ satisfy

$$
\int_{a_{1}}^{b_{1}} g_{1}(x) d x=1-\alpha \text { and } a_{1} g_{1}\left(a_{1}\right)=b_{1} g_{1}\left(b_{1}\right) .
$$

We aim at constructing intervals of smaller ratio of endpoints that, in addition, maintain coverage probability above the nominal level $1-\alpha$. In typical cases (such as Examples 1-3 in Section 2), the problem of interval estimation of $\sigma_{1}$ subject to the restriction $\sigma_{1} \leq \sigma_{2}$ is invariant under the group of transformations $\left(T_{1}, S_{1}, T_{2}, S_{2}\right)$ $\longrightarrow\left(c T_{1}, c S_{1}, c T_{2}, c S_{2}\right), c>0$, and the class of equivariant intervals is $\mathcal{C}=$ $\left\{\left(\psi_{1}^{*}\left(S_{2} / S_{1}, T_{1} / S_{1}, T_{2} / S_{1}\right) S_{1}, \psi_{2}^{*}\left(S_{2} / S_{1}, T_{1} / S_{1}, T_{2} / S_{1}\right) S_{1}\right): \psi_{1}^{*}<\psi_{2}^{*}\right.$ positive functions $\}$. For $V=S_{2} / S_{1}$ we first consider the subclass of intervals of the form $I=$ $\left(\psi_{1}(V) S_{1}, \psi_{2}(V) S_{1}\right)$. For $v>0$, we find $\psi_{10}=\psi_{10}(v)$ and $\psi_{20}=\psi_{20}(v)$ so that

$\int_{1 / \psi_{20}}^{1 / \psi_{10}} x g_{1}(x) g_{2}(x v) d x=\int_{a_{1}}^{b_{1}} x g_{1}(x) g_{2}(x v) d x$ and $\frac{1}{\psi_{10}^{2}} g_{1}\left(\frac{1}{\psi_{10}}\right) g_{2}\left(\frac{v}{\psi_{10}}\right)=\frac{1}{\psi_{20}^{2}} g_{1}\left(\frac{1}{\psi_{20}}\right) g_{2}\left(\frac{v}{\psi_{20}}\right)$.

As in (2.4), the existence and uniqueness of $\psi_{10}$ and $\psi_{20}$ follows from the continuity of $x g_{1}(x) g_{2}(x v)$ and the unimodality of $x^{2} g_{1}(x) g_{2}(x v)$ as functions of $x>0$ upon using Lemma 4.1 and (A1). We then define a confidence interval for $\sigma_{1}$ by

$$
I\left(S_{1}, V\right)=\left(\psi_{1}(V) S_{1}, \psi_{2}(V) S_{1}\right),
$$

where

$$
\psi_{1}(V)=\min \left\{\psi_{10}(V), 1 / b_{1}\right\} \text { and } \psi_{2}(V)=\min \left\{\psi_{20}(V), 1 / a_{1}\right\} .
$$

Theorem 3.1 Assume that (A1) holds. If $\sigma_{1} \leq \sigma_{2}$ and

$$
\mathrm{P}\left(b_{1} g_{2}\left(b_{1} V\right)>a_{1} g_{2}\left(a_{1} V\right)\right)>0
$$

then we have the following.

(i) $\mathrm{P}\left(\psi_{1}(V)<1 / b_{1}\right)>0$.

(ii) $\mathrm{P}\left(\sigma_{1} \in I\left(S_{1}, V\right)\right) \geq \mathrm{P}\left(\sigma_{1} \in I_{1}\right)=1-\alpha$ and the inequality is strict unless $\sigma_{1}=\sigma_{2}$.

(iii) The ratio of endpoints of $I\left(S_{1}, V\right)$ is less than or equal to $b_{1} / a_{1}$ and is strictly less with positive probability. 
Proof. (i) The second equality in (3.3), the unimodality of $x^{2} g_{1}(x) g_{2}(x v)$ as a function of $x$, and an argument analogous to that in the beginning of the proof of Theorem 2.2 yield $\left\{v>0: \psi_{1}(v)<1 / b_{1}\right\}=\left\{v>0: b_{1} g_{2}\left(b_{1} v\right)>a_{1} g_{2}\left(a_{1} v\right)\right\}$. Thus, the result follows from (3.5).

(ii), (iii) The conditional density of $S_{1} / \sigma_{1}$ given $V=v$ is $g_{1}\left(x \mid v ; \sigma_{1}, \sigma_{2}\right) \propto x g_{1}(x) g_{2}\left(\frac{\sigma_{1}}{\sigma_{2}} x v\right)$ and thus for $\sigma_{1}=\sigma_{2}$ it simplifies to $g_{1}(x \mid v) \propto x g_{1}(x) g_{2}(x v)$. Furthermore, (A1) and the relation $\sigma_{1} \leq \sigma_{2}$ imply that $g_{1}\left(x \mid v ; \sigma_{1}, \sigma_{2}\right) / g_{1}(x \mid v)$ is strictly increasing in $x>0$ unless $\sigma_{1}=\sigma_{2}$. The proof of the result is now as that of Theorem 2.2 replacing $W_{1}, a, b, f_{1}\left(x \mid w_{1}\right)$, and $f_{1}\left(x \mid w_{1} ; \mu_{1}, \sigma_{1}\right)$ by $V, a_{1}, b_{1}, g_{1}(x \mid v)$, and $g_{1}\left(x \mid v ; \sigma_{1}, \sigma_{2}\right)$ respectively.

For further improvement we study intervals in the larger class $\mathcal{C}$, which, in addition, employ $W_{1}=T_{1} / S_{1}$ and $Z_{1}=T_{2} / S_{1}$, i.e., $I=\left(\psi_{1}^{*}\left(V, W_{1}, Z_{1}\right) S_{1}, \psi_{2}^{*}\left(V, W_{1}, Z_{1}\right) S_{1}\right)$. We let $v>0, w_{1}>0, z_{1}>0$ and determine $\psi_{10}^{*}=\psi_{10}^{*}\left(v, w_{1}, z_{1}\right), \psi_{20}^{*}=\psi_{20}^{*}\left(v, w_{1}, z_{1}\right)$ so that

$$
\int_{1 / \psi_{20}^{*}}^{1 / \psi_{10}^{*}} x^{3} g_{1}(x) g_{2}(x v) h_{1}\left(x w_{1}\right) h_{2}\left(x z_{1}\right) d x=\int_{1 / \psi_{2}}^{1 / \psi_{1}} x^{3} g_{1}(x) g_{2}(x v) h_{1}\left(x w_{1}\right) h_{2}\left(x z_{1}\right) d x
$$

and

$$
\frac{1}{\psi_{10}^{*}} g_{1}\left(\frac{1}{\psi_{10}^{*}}\right) g_{2}\left(\frac{v}{\psi_{10}^{*}}\right) h_{1}\left(\frac{w_{1}}{\psi_{10}^{*}}\right) h_{2}\left(\frac{z_{1}}{\psi_{10}^{*}}\right)=\frac{1}{\psi_{20}^{*}} g_{1}\left(\frac{1}{\psi_{20}^{*}}\right) g_{2}\left(\frac{v}{\psi_{20}^{*}}\right) h_{1}\left(\frac{w_{1}}{\psi_{20}^{*}}\right) h_{2}\left(\frac{z_{1}}{\psi_{20}^{*}}\right),
$$

where $\psi_{1}=\psi_{1}(v)$ and $\psi_{2}=\psi_{2}(v)$ are as in (3.4). Setting

$$
\begin{aligned}
& \psi_{1}^{*}\left(v, w_{1}, z_{1}\right)= \begin{cases}\min \left\{\psi_{10}^{*}\left(v, w_{1}, z_{1}\right), \psi_{1}(v)\right\} & , \text { if } v>0, w_{1}>0, z_{1}>0, \\
\psi_{1}(v) & , \text { otherwise }\end{cases} \\
& \psi_{2}^{*}\left(v, w_{1}, z_{1}\right)= \begin{cases}\min \left\{\psi_{20}^{*}\left(v, w_{1}, z_{1}\right), \psi_{2}(v)\right\} & , \text { if } v>0, w_{1}>0, z_{1}>0, \\
\psi_{2}(v) & , \text { otherwise }\end{cases}
\end{aligned}
$$

we obtain the interval

$$
I\left(S_{1}, V, W_{1}, Z_{1}\right)=\left(\psi_{1}^{*}\left(V, W_{1}, Z_{1}\right) S_{1}, \psi_{2}^{*}\left(V, W_{1}, Z_{1}\right) S_{1}\right) .
$$

The next result establishes the domination of $I\left(S_{1}, V, W_{1}, Z_{1}\right)$ over $I\left(S_{1}, V\right)$ and hence over $I_{1}$.

Theorem 3.2 Assume that (A1), (A2), (A3), (A'2), (A'3), and (C1) hold. If $\sigma_{1} \leq \sigma_{2}$ and

$$
\begin{aligned}
\mathrm{P}\left(W_{1}>0, Z_{1}>0, b_{1} g_{2}\left(b_{1} V\right)>a_{1} g_{2}\left(a_{1} V\right),\right. \\
\left.\frac{1}{\psi_{1}^{2}(V)} h_{1}\left(\frac{W_{1}}{\psi_{1}(V)}\right) h_{2}\left(\frac{Z_{1}}{\psi_{1}(V)}\right)>\frac{1}{\psi_{2}^{2}(V)} h_{1}\left(\frac{W_{1}}{\psi_{2}(V)}\right) h_{2}\left(\frac{Z_{1}}{\psi_{2}(V)}\right)\right)>0,
\end{aligned}
$$

where $\psi_{1}(V)$ and $\psi_{2}(V)$ are as in (3.4) then we have the following.

(i) $\mathrm{P}\left(\psi_{10}^{*}\left(V, W_{1}, Z_{1}\right)<\psi_{1}(V)\right)>0$. 
(ii) $\mathrm{P}\left(\sigma_{1} \in I\left(S_{1}, V, W_{1}, Z_{1}\right)\right) \geq \mathrm{P}\left(\sigma_{1} \in I\left(S_{1}, V\right)\right) \geq 1-\alpha$.

(iii) The ratio of endpoints of $I\left(S_{1}, V, W_{1}, Z_{1}\right)$ is less than or equal to that of $I\left(S_{1}, V\right)$ and is strictly less with positive probability.

Proof. (i) Using (3.3), (3.4), the relations defining $\psi_{10}^{*}, \psi_{20}^{*}$, the unimodality of $x^{4} g_{1}(x) g_{2}(x v) h_{1}\left(x w_{1}\right) h_{2}\left(x z_{1}\right)$, and the argument in the beginning of the proof of Theorem 2.2 we obtain that $\left\{v>0, w_{1}>0, z_{1}>0: \psi_{10}^{*}\left(v, w_{1}, z_{1}\right)<\psi_{1}(v)\right\}=$ $\left\{v>0, w_{1}>0, z_{1}>0: b_{1} g_{2}\left(b_{1} v\right)>a_{1} g_{2}\left(a_{1} v\right)\right.$,

Thus, the result follows from (3.8).

$$
\left.\frac{1}{\psi_{1}^{2}(v)} h_{1}\left(\frac{w_{1}}{\psi_{1}(v)}\right) h_{2}\left(\frac{z_{1}}{\psi_{1}(v)}\right)>\frac{1}{\psi_{2}^{2}(v)} h_{1}\left(\frac{w_{1}}{\psi_{2}(v)}\right) h_{2}\left(\frac{z_{1}}{\psi_{2}(v)}\right)\right\} .
$$

(ii), (iii) The conditional density of $S_{1} / \sigma_{1}$ given $V=v>0, W_{1}=w_{1}>0$, and $Z_{1}=z_{1}>0$ is $g_{1}\left(x \mid v, w_{1}, z_{1} ; \mu_{i}, \sigma_{i}\right) \propto$

$$
x^{3} g_{1}(x) g_{2}\left(\frac{\sigma_{1}}{\sigma_{2}} x v\right) h_{1}\left(x w_{1} ; \mu_{1}, \sigma_{1}\right) h_{2}\left(\frac{\sigma_{1}}{\sigma_{2}} x z_{1} ; \mu_{2}, \sigma_{2}\right) I_{\left(\max \left(0, \frac{\lambda_{1}}{w_{1}}, \frac{\lambda_{2} \sigma_{2}}{z_{1} \sigma_{1}}\right), \infty\right)}(x)
$$

which for $\mu_{i}=\mu_{i 0}$ and $\sigma_{i}=\sigma_{i 0}, i=1,2$, simply becomes

$$
g_{1}\left(x \mid v, w_{1}, z_{1}\right) \propto x^{3} g_{1}(x) g_{2}(x v) h_{1}\left(x w_{1}\right) h_{2}\left(x z_{1}\right) I_{(0, \infty)}(x) .
$$

Thus (A1) to (C1) imply that $g_{1}\left(x \mid v, w_{1}, z_{1} ; \mu_{i}, \sigma_{i}\right) / g_{1}\left(x \mid v, w_{1}, z_{1}\right)$ is nondecreasing in $x>0$. Furthermore, when $b_{1} g_{2}\left(b_{1} v\right)>a_{1} g_{2}\left(a_{1} v\right)$ we have $\psi_{1}(v)=\psi_{10}(v)$ (see the proof of part $i$ of Theorem 3.1). Hence, by (3.3), the last inequality in (3.8) can be written as $\frac{1}{\psi_{1}} g_{1}\left(\frac{1}{\psi_{1}} \mid v, w_{1}, z_{1}\right)>\frac{1}{\psi_{2}} g_{1}\left(\frac{1}{\psi_{2}} \mid v, w_{1}, z_{1}\right)$. In other words, (3.8) is equivalent to $\mathrm{P}\left(W_{1}>0, Z_{1}>0, b_{1} g_{2}\left(b_{1} V\right)>a_{1} g_{2}\left(a_{1} V\right)\right.$,

$$
\left.\frac{1}{\psi_{1}(V)} g_{1}\left(\frac{1}{\psi_{1}(V)} \mid V, W_{1}, Z_{1}\right)>\frac{1}{\psi_{2}(V)} g_{1}\left(\frac{1}{\psi_{2}(V)} \mid V, W_{1}, Z_{1}\right)\right)>0 .
$$

The rest of the proof proceeds as in Theorem 2.2 replacing $a, b, f_{1}\left(x \mid w_{1}\right)$ and $f_{1}\left(x \mid w_{1} ; \mu_{1}, \sigma_{1}\right)$ by $1 / \psi_{1}, 1 / \psi_{2}, g_{1}\left(x \mid v, w_{1}, z_{1}\right)$, and $g_{1}\left(x \mid v, w_{1}, z_{1} ; \mu_{i}, \sigma_{i}\right)$ respectively.

To illustrate Conditions (3.5) and (3.8), we consider the case where the $g_{i}^{\prime} s$ and $h_{i}^{\prime} s$ are the gamma densities in Theorem 2.3. Using the relations defining $a_{1}$ and $b_{1}$ in (3.2), it is straightforward to show that (3.5) is equivalent to $\mathrm{P}\left(V<\frac{\alpha_{2} \beta_{2}}{\alpha_{1} \beta_{1}}\right)>0$ which clearly holds. Also, using (3.3), it can be seen that (3.8) is equivalent to

$$
\mathrm{P}\left(W_{1}>0, Z_{1}>0, V<\frac{\alpha_{2} \beta_{2}}{\alpha_{1} \beta_{1}}, \frac{W_{1}}{\delta_{1}}+\frac{Z_{1}}{\delta_{2}}<\frac{\gamma_{1}+\gamma_{2}}{\alpha_{1}+\alpha_{2}}\left(\frac{1}{\beta_{1}}+\frac{V}{\beta_{2}}\right)\right)>0
$$

which is satisfied. Thus, in particular, Conditions (3.5) and (3.8) hold for the normal, exponential, and inverse Gaussian models in Examples 1-3 of Section 2.

Remark 3.1. We comment on the computation of the improved intervals $I\left(S_{1}, V\right)$ and $I\left(S_{1}, V, W_{1}, Z_{1}\right)$ when the $g_{i}^{\prime} \mathrm{s}$ and $h_{i}^{\prime}$ s are the gamma densities in Theorem 2.3. In this case, making an obvious scale transformation, the integrand in (3.3) becomes the gamma density with shape parameter $\alpha_{1}+\alpha_{2}$ and scale parameter $\beta_{1}$. Similarly, the integrand in (3.6) becomes the gamma density with shape parameter $\alpha_{1}+\alpha_{2}+\gamma_{1}+\gamma_{2}$ and scale $\beta_{1}$. On the other hand, the computation of $I_{1}$ in (3.1) involves the gamma density 
with shape parameter $\alpha_{1}$ and scale $\beta_{1}$. Therefore, the main steps for the computation of $I\left(S_{1}, V\right)$ and $I\left(S_{1}, V, W_{1}, Z_{1}\right)$ are, merely, reruns of the algorithm that produces the standard interval $I_{1}$ in (3.1). Thus, the computational difficulty of all three intervals is essentially the same.

Remark 3.2. It is clear from the proofs of Theorems 3.1 and 3.2 that the standard interval $I_{1}$ in (3.1) can be improved by choosing any subset of $V, W_{1}, Z_{1}$. If, initially, one uses only $W_{1}$ the resulting improvement is also a standard interval in the sense that its construction does not exploit the restriction $\sigma_{1} \leq \sigma_{2}$. Then better improvements can be obtained by employing in addition $V$ and/or $Z_{1}$.

We now present an application of the above results. Consider the balanced one way random effects linear model $Y_{i j}=\mu+\alpha_{i}+e_{i j}, i=1, \ldots, \kappa, j=1, \ldots, m$, where $\alpha_{i}$ 's and $e_{i j}$ 's are independent random variables with distributions $\alpha_{i} \sim N\left(0, \sigma_{0}\right)$ and $e_{i j} \sim$ $N\left(0, \sigma_{1}\right)$. The minimal sufficient statistic $\left(S_{1}, S_{2}, \bar{Y}\right)$ has independent components $S_{1}=$ $\sum_{i=1}^{\kappa} \sum_{j=1}^{m}\left(Y_{i j}-\bar{Y}_{i}\right)^{2} \sim \sigma_{1} \chi_{n}^{2}, S_{2}=m \sum_{i=1}^{\kappa}\left(\bar{Y}_{i}-\bar{Y}\right)^{2} \sim\left(\sigma_{1}+m \sigma_{0}\right) \chi_{\kappa-1}^{2}, \bar{Y}=\sum_{i=1}^{\kappa} \sum_{j=1}^{m} Y_{i j} /(m \kappa) \sim$ $N\left(\mu, \frac{\sigma_{1}+m \sigma_{0}}{m \kappa}\right)$, where $\bar{Y}_{i}=\sum_{j=1}^{m} Y_{i j} / m$ and $n=\kappa(m-1)$. The standard interval for $\sigma_{1}$ is based on $S_{1}$ whereas better ones are given in Kubokawa et al. [15]. The latter improve on coverage probability while maintaining the same ratio of endpoints as the standard interval. Setting $\sigma_{2}=\sigma_{1}+m \sigma_{0}$ and $T_{2}=m \kappa \bar{Y}^{2} \sim \sigma_{2} \chi_{1}^{2}\left(\frac{m \kappa \mu^{2}}{\sigma_{2}}\right)$, Theorems 3.1 and 3.2 can be used to provide improvements in terms of both coverage probability and ratio of endpoints by employing $S_{2} / S_{1}$ and/or $m \kappa \bar{Y}^{2} / S_{1}$. The magnitude of the improvement of $I_{1}\left(S_{1}, V\right)$ over $I_{1}$ (for the above model) was investigated numerically for selected values of $\kappa, m$ and nominal level $1-\alpha=0.95$. In Figures 4 and 5 , the coverage probability and percentage improvement in expected ratio of endpoints are plotted against $\sigma_{0}$. (Note that these quantities are functions of $\sigma_{2} / \sigma_{1}=1+m \sigma_{0} / \sigma_{1}$, so $\sigma_{1}$ was taken one.) The numerical results indicate that $I_{1}\left(S_{1}, V\right)$ performs better, in terms of maximum improvement, when the number of levels of the random effect is large relative to the cell size.

Theorems 3.1 and 3.2 can be extended towards two directions: one is to treat more than two populations and the other one is to allow $T_{j}$ to have a scale parameter $\beta_{j}$ rather than $\sigma_{j}$. Specifically, consider the model where one observes independent random variables $S_{1}, \ldots, S_{\kappa}$ and $T_{1}, \ldots, T_{m}$ such that $S_{i} / \sigma_{i} \sim g_{i}(x) I_{(0, \infty)}(x)$ and $T_{j} / \beta_{j} \sim h_{j}\left(x ; \mu_{j}, \beta_{j}\right) I_{\left(\lambda_{j}, \infty\right)}(x)$. Then under the restrictions $\sigma_{1} \leq \sigma_{i}, i=2, \ldots, \kappa$, $\sigma_{1} \leq \beta_{j}, j=1, \ldots, m$ and some obvious modifications of (A1) to (C1), one can derive improved intervals for $\sigma_{1}$ which are extensions of those in Theorems 3.1 and 3.2. An interesting situation where the above applies is the problem of interval estimation of the error variance in a general balanced mixed linear model. This model is studied in Mathew and Sinha [21] and Mathew et al. [22], where the latter presents improved point estimators of the error variance, $\sigma_{1}$ say. Improved intervals for $\sigma_{1}$, however, are 
not available in the literature. The model can be described by means of independent canonical variables $S_{i} \sim \sigma_{i} \chi_{q_{i}}^{2}$ and $T_{j} \sim \beta_{j} \chi_{p_{j}}^{2}\left(\delta_{j}\right)$ where $\sigma_{1} \leq \sigma_{i}$ and $\sigma_{1} \leq \beta_{j}$ for $i=2, \ldots, \kappa$ and $j=1, \ldots, m$. (For instance, $S_{1}$ is the error sum of squares.) We refer to the above articles for a detailed account on this canonical form of the model. Thus, the interval estimation of $\sigma_{1}$ falls into the above framework and improved procedures can be produced.

We close this section by noting that the standard minimum ratio of endpoints interval for $\sigma_{2}$, which is of the form $\left(S_{2} / b_{2}, S_{2} / a_{2}\right)$, can be improved (under $\sigma_{1} \leq \sigma_{2}$ ) by an interval of the form $\left(\phi_{1}(S) S_{2}, \phi_{2}(S) S_{2}\right)$. The construction of the latter is analogous to that in Theorem 3.1. Details of this construction are given in Iliopoulos and Kourouklis [8].

\section{Appendix}

Lemma 4.1 Let $g(x)$ be a density function on $(0, \infty)$ with $g(x)>0$ for every $x>0$ and such that

$$
g\left(c_{1} x\right) / g\left(c_{2} x\right) \text { is strictly increasing in } x \text { for } 0<c_{1}<c_{2} .
$$

Then $g(x)$ is continuous on $(0, \infty), x g(x)$ is unimodal, and $\lim _{x \rightarrow 0} x g(x)=\lim _{x \rightarrow \infty} x g(x)=0$.

Proof. Condition (4.1) means that the scale family $\left\{c^{-1} g(x / c): c>0\right\}$ has the monotone likelihood ratio property and hence that $-\log \left(e^{y} g\left(e^{y}\right)\right)$ is a convex function of $y \in(-\infty, \infty)$, cf. Lehmann [18], p.510. Consequently, $g(x)$ is continuous on $(0, \infty)$. In addition, $f(y)=e^{y} g\left(e^{y}\right)$ is either monotone or increasing and then decreasing. The former obviously cannot hold since $f(y)$ is a strictly positive density on $(-\infty, \infty)$. Therefore, for $x>0, x g(x)=f(\log x)$ is increasing and then decreasing function of $x$. In addition, $x g(x)$ cannot be constant on any interval $\left(x_{1}, x_{2}\right)$, since if it were then setting $x_{0}=\left(x_{1} x_{2}\right)^{1 / 2}$ and $c_{2}=x_{0} / x_{1}$ we would have $g(x) / g\left(c_{2} x\right)=c_{2}$ for all $x \in\left(x_{1}, x_{0}\right)$ which contradicts (4.1). Now for small $x, x g(x)<\int_{x}^{2 x} g(v) d v$, implying $\lim _{x \rightarrow 0} x g(x)=0$. Similarly for large $x, x g(x)<2 \int_{x / 2}^{x} g(v) d v$, and thus $\lim _{x \rightarrow \infty} x g(x)=0$.

Lemma 4.2 Suppose that $b_{1}(x)$ and $b_{2}(x)$ are densities supported on the same interval of the real line and $b_{2}(x) / b_{1}(x)$ is strictly increasing. If $X$ is a random variable with density $b_{1}(x)$ or $b_{2}(x)$ and $h(x)$ is nondecreasing but not constant on the common support of $b_{1}(x)$ and $b_{2}(x)$, then $E_{b_{2}} h(X)>E_{b_{1}} h(X)$ (provided expectations exist).

Proof. It is given in Lehmann [18], p.85.

Lemma 4.3 Let $X_{i}$ and $Y$ be independent random variables, where $X_{i}$ has density $f_{i}(x), i=1,2, Y$ has density $f(x), X_{1}, Y$ are supported on $(0, \infty)$ and $X_{2}$ is supported on $(\mu, \infty), \mu \geq 0$. Furthermore suppose that $f_{2}(x) / f_{1}(x)$ is nondecreasing on $(0, \infty)$ and $f\left(c_{1} x\right) / f\left(c_{2} x\right)$ is strictly increasing for $0<c_{1}<c_{2}$. If $h_{i}(x)$ is the density of $X_{i} / Y, i=1,2$, then $h_{2}(x) / h_{1}(x)$ is strictly increasing in $x>0$ unless $f_{1}=f_{2}$. 
Proof. It can be given using arguments of total positivity (Karlin [11], composition theorem) or variation diminishing transformations (Brown et al. [1]). See also Iliopoulos and Kourouklis [10] for a direct proof.

Lemma 4.4 Let $Y_{i}, i=1,2$, be independent random variables with densities $g_{i}(x)$, respectively, supported on $(0, \infty)$. Suppose that $g_{i}\left(c_{1} x\right) / g_{i}\left(c_{2} x\right)$ is strictly increasing in $x>0$ for $0<c_{1}<c_{2}$ and denote by $g(x)$ the density of $Y_{1} / Y_{2}$. Then $g\left(c_{1} x\right) / g\left(c_{2} x\right)$ is also strictly increasing in $x>0$ for $0<c_{1}<c_{2}$.

Proof. It is an application of Lemma 4.3 with $X_{1}=Y_{1} / c_{2}, X_{2}=Y_{1} / c_{1}$, and $Y=Y_{2}$.

Lemma 4.5 Let $f(x)$ be a positive valued function on $(0, \infty)$ such that $x f(x)$ is unimodal and $c f(c)=d f(d)$ for $0<c<d$. Suppose also that for $0<a<b$, neither of the intervals $(a, b)$ and $(c, d)$ is a proper subset of the other. Then the relation $b f(b)>($ resp. $<)$ af $(a)$ implies $d>($ resp. $<) b$.

Proof. The argument is along the lines of that in the proof of Lemma A.2 of Goutis and Casella [5].

Lemma 4.6 Suppose that $f_{1}(x)$ is an integrable function on $(0, \infty)$ such that $f_{1}(x)>0$ for every $x>0$ and, for $\mu \geq 0, f_{2}(x)$ is an integrable function on $(\mu, \infty)$. Furthermore assume that for constants $a<b$ and $c<d$ in the interval $(\mu, \infty)$ we have

$$
\int_{c}^{d} f_{1}(x) d x=\int_{a}^{b} f_{1}(x) d x
$$

If $d>b$ and $f_{2}(x) / f_{1}(x)$ is strictly increasing (resp. nondecreasing) in $(\mu, \infty)$ or if $d<b$ and $f_{2}(x) / f_{1}(x)$ is strictly decreasing (resp. nonincreasing) in $(\mu, \infty)$, then

$$
\int_{c}^{d} f_{2}(x) d x>(\text { resp. } \geq) \int_{a}^{b} f_{2}(x) d x
$$

Proof. We prove the result only when $d>b$ and $f_{2}(x) / f_{1}(x)$ is strictly increasing. Then, by (4.2), the possible cases are:

(i) $a<c<b<d$,

(ii) $a<b \leq c<d$.

Assume that (i) holds. Then $\int_{c}^{d} f_{2}(x) d x-\int_{a}^{b} f_{2}(x) d x=\int_{b}^{d} f_{2}(x) d x-\int_{a}^{c} f_{2}(x) d x>$ $\frac{f_{2}(b)}{f_{1}(b)} \int_{b}^{d} f_{1}(x) d x-\frac{f_{2}(c)}{f_{1}(c)} \int_{a}^{c} f_{1}(x) d x=\left(\frac{f_{2}(b)}{f_{1}(b)}-\frac{f_{2}(c)}{f_{1}(c)}\right) \int_{a}^{c} f_{1}(x) d x>0$. The proof under (ii) is similar. 

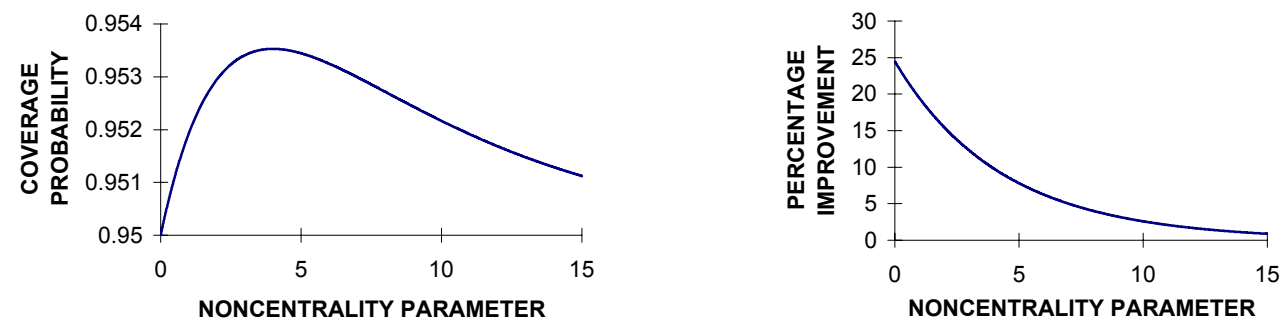

Fig.1. $n=m=3, p=3$. Maximum coverage probability 0.9535 . Maximum percentage improvement in expected ratio of endpoints $24.5 \%$.
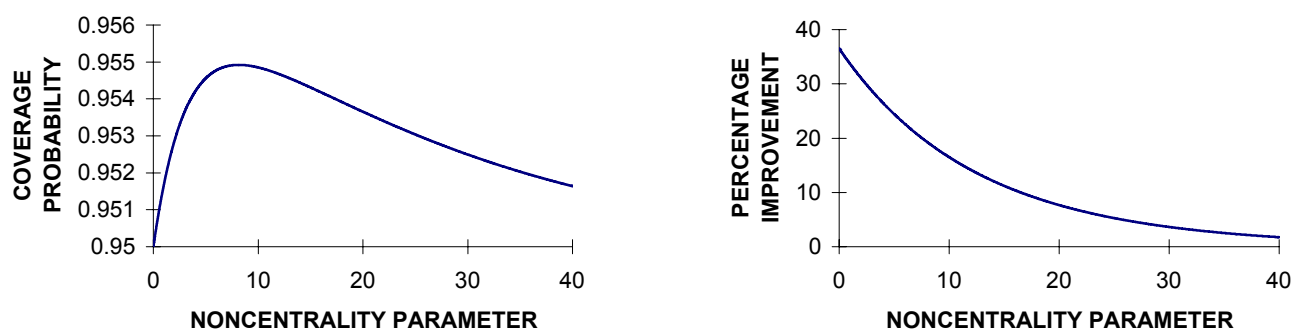

Fig.2. $n=m=3, p=10$. Maximum coverage probability 0.9549. Maximum percentage improvement in expected ratio of endpoints $36.5 \%$.
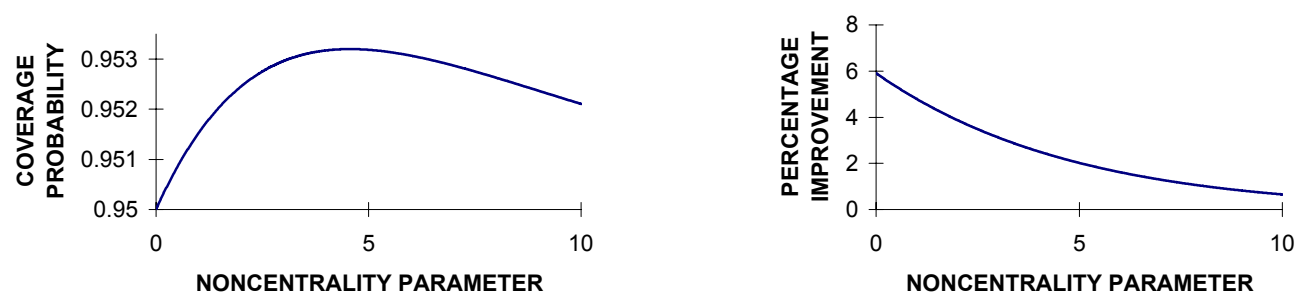

Fig.3. $n=20, m=30, p=10$. Maximum coverage probability 0.9532 . Maximum percentage improvement in expected ratio of endpoints $5.91 \%$.
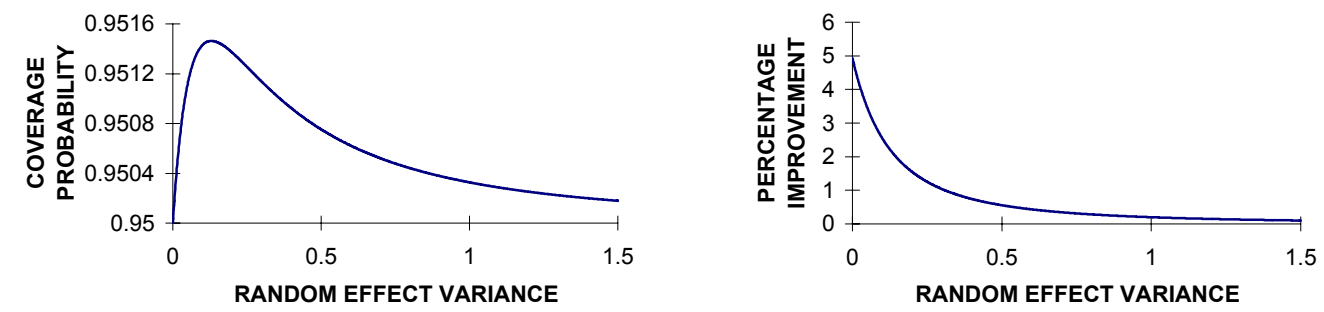

Fig.4. $k=5, m=6, n=\kappa(m-1)=25$. Maximum coverage probability 0.9515 . Maximum percentage improvement in expected ratio of endpoints $4.93 \%$.
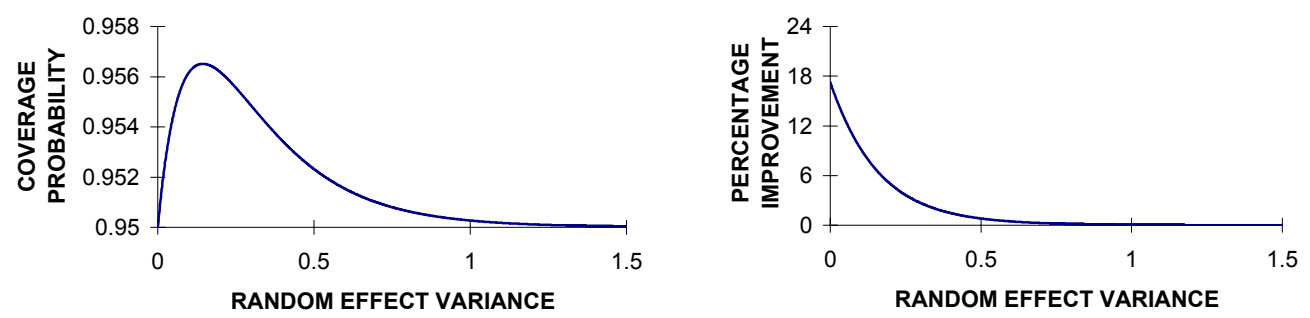

Fig.5. $k=25, m=2, n=\kappa(m-1)=25$. Maximum coverage probability 0.9565 . Maximum percentage improvement in expected ratio of endpoints $17.25 \%$. 


\section{References}

[1] Brown, L. D., Johnstone, I. M. and MacGibbon, B. (1981). Variation diminishing transformations: a direct approach to total positivity and its statistical applications. J. Amer. Statist. Association, 76, 824-832.

[2] Cohen, A. and Sackrowitz, H. B. (1996). Lower confidence regions for restricted parameter spaces. Math. Methods of Statist., 5, 113-123.

[3] Gelfand, A. E. and Dey, D. K. (1988). On the estimation of a variance ratio. J. Statist. Plann. Inference, 19, 121-131.

[4] Ghosh, M. and Kundu, S. (1996). Decision theoretic estimation of the variance ratio. Statist. Decisions, 14, 161-175.

[5] Goutis, C. and Casella, G. (1991). Improved invariant confidence intervals for a normal variance. Ann. Statist., 19, 2015-2031.

[6] Goutis, C. and Casella, G. (1995). Improved invariant set estimation for general-scale families. J. Statist. Plann. Inference, 44, 327-340.

[7] Hwang, J. T. G. and Peddada, S. D. (1994). Confidence interval estimation subject to order restrictions. Ann. Statist., 22, 67-93.

[8] Iliopoulos, G. and Kourouklis, S. (1997). Interval estimation for the ratio of scale parameters and for ordered scale parameters. Technical Report, TR 9/97, Dept. of Math. \& Stat., Univ. of Cyprus.

[9] Iliopoulos, G. and Kourouklis, S. (1998). On improved interval estimation for the generalized variance. J. Statist. Plann. Inference, 66, 305-320.

[10] Iliopoulos, G. and Kourouklis, S. (1999). Improving on the best affine equivariant estimator of the ratio of generalized variances. J. Multivariate Anal., 68, 176-192.

[11] Karlin, S. (1968). Total positivity, Vol.1, Stanford Univ. Press, Stanford, CA.

[12] Kourouklis, S. (1997). A new property of the inverse Gaussian distribution with applications. Statist. Probab. Letters, 32, 161-166.

[13] Kubokawa, T. (1994). Double shrinkage estimation of ratio of scale parameters. Ann. Inst. Statist. Math., 46, 95-116.

[14] Kubokawa, T. and Saleh, A.K.Md.E. (1994). Estimation of location and scale parameters under order restrictions. J. Statistical Research, 28, 41-51.

[15] Kubokawa, T., Saleh, A.K.Md.E. and Makita, S. (1993). On improved positive estimators of variance components. Statist. Decisions, 11, 1-16.

[16] Kubokawa, T. and Srivastava, M. S. (1996). Double shrinkage estimators of ratio of variances..In Multidimensional Statistical Analysis and Theory of Random Matrices, ed. A.K. Gupta and V.L. Girko. 139-154, VSP, Netherlands.

[17] Kushary, D. and Cohen, A. (1989). Estimating ordered location and scale parameters. Statist. Decisions, 7, 201-213.

[18] Lehmann, E. L. (1986). Testing Statistical Hypotheses, 2nd ed., Springer, New York. 
[19] Madi, T. M. (1995). On the invariant estimation of a normal variance ratio. J. Statist. Plann. Inference, 44, 349-357.

[20] Madi, T. M. and Tsui, K. W. (1990). Estimation of the ratio of the scale parameters of two exponential distributions with unknown location parameters. Ann. Inst. Statist. Math., 42, $77-87$.

[21] Mathew, T. and Sinha, B.K. (1988). Optimum tests for fixed effects and variance components in balanced models. J. Amer. Statist. Assoc., 83, 133-135.

[22] Mathew, T., Sinha, B.K., and Sutradhar, B. (1992). Improved estimation of error variance in general balanced mixed models. Statist. Decisions, 10, 227-238.

[23] Nagata, Y. (1989). Improvements of interval estimations for the variance and the ratio of two variances. J. Japan Statist. Soc., 19, 151-161.

[24] Robertson, T., Wright, F.T. and Dykstra, R.L. (1998). Order restricted statistical inference. Wiley, New York.

[25] Stein, C. (1964). Inadmissibility of the usual estimator for the variance of a normal distribution with unknown mean. Ann. Inst. Statist. Math., 16, 155-160.

[26] van Eeden, C. (1956). Maximum likelihood estimation of ordered probabilities. Proc. Kon. Nederl. Akad. Wetensch, A60, 444-455.

[27] van Eeden, C. (1957a). Maximum likelihood estimation of partially or completely ordered parameters. Proc. Kon. Nederl. Akad. Wetensch, A60, 128-136 and 201-211.

[28] van Eeden, C. (1957b). Note on two methods of estimating ordered parameters of probability distributions. Proc. Kon. Nederl. Akad. Wetensch, A60, 506-512.

[29] van Eeden, C. (1957c). A least squares inequality for maximum likelihood estimates of ordered parameters. Proc. Kon. Nederl. Akad. Wetensch, A60, 513-521.

[30] van Eeden, C. (1958). Testing and estimating ordered parameters of probability distributions. Ph.D. thesis, Univ. of Amsterdam.

[31] van Eeden, C. (1996). Estimation in restricted parameter spaces - some history and some recent developments. CWI Quarterly, 9, 69-76.

George Iliopoulos

University of Patras

Department of Mathematics

26500 Rio, Patras

Greece
Stavros Kourouklis

University of Patras

Department of Mathematics

26500 Rio, Patras

Greece 